\title{
A UTILIZAÇÃO DO BRINQUEDO NA SALA DE RECUPERAÇÃO: UM RECURSO A MAIS PARA ASSISTÊNCIA DE ENFERMAGEM Á CRIANC̣A
}

\author{
Êrica Rosalba Mallmann Duarte ${ }^{1}$ \\ Ana Maria Müller² \\ Sônia Maria Alexandre Bruno ${ }^{3}$ \\ Ana Lúcia Saldanha Duarte ${ }^{4}$
}

\begin{abstract}
RESUMO. Trata-se de um estudo preliminar sobre a experiência dos enfermeiros e psicólogos que atuam junto às crianças na Unidade de Centro Cirúrgico do Hospital de Clínicas de Porto Alegre quando introduziram brinquedos na Sala de Recuperação com o objetivo de diminuir as reações de desconforto apresentadas pela criança durante sua recuperação. Procuramos neste trabalho estudar a influência do brinquedo no processo de recuperação de crianças no pós-cirúrgico bem como mostrar aos profissionais que atuam nesta área a necessidade de busca de recursos que contribuem para o aprimoramento da assistência de enfermagem às crinças.
\end{abstract}

\begin{abstract}
Nurse and Psichologists who work the children in the surgical unit of Hospital de Clínicas de Porto Alegre have brought toys in thepost surgical care ;oom in order to miniminise children's discomfort during their recuperation of anaesthesia and surgery. The purpose of this study is to show the importance of toys on children's post surgical recuperation and to show professionals of this area the necessity of constant search of resources which can contribute to a better nursing assistance to children.
\end{abstract}

\section{INTRODUC̣ÃO}

"Proporcionar brinquedos para a criança indica que sua necessidade de trabalho e de brinquedo foi entendida e lhe assegura que embora a situação presente seja confusa e dolorosa ela pode encontrar amizade e consideração" (WAETCHER, 1979).

A enfermagem deve ser entendida como a ciência que assiste ao indivíduo e família, doente ou sadio, na satisfação de suas necessidades básicas que são comuns a todos os seres humanos, crianças ou adultos. Estas necessidades podem ser fisiológicas, de segurança, de espaço pessoal, de reconhecimento, de estima, de afeto, de criar, de conhecimento, de compreensão e de estética, entre outros.

Quando a assistência de enfermagem é dirigida à criança e a sua família, o enfermeiro deve estar consciente da necessidade de "compreensão" da criança como um ser em desenvolvimento, com modos peculiares de sentir e de pensar, e com métodos individuais de lutar com o ambiente (WAETCHER, 1979).

Atualmente torna-se evidente a preocupação dos profissionais de saúde no sentido de assistir à criança e à família, em situação de doença, de modo a minimizar os efeitos negativos de um trauma devido a sepa-

\footnotetext{
1 Professora Assistente da Escola de Enfermagem da Universidade Federal do Rio Grande do Sul, Chefe do Serviço de Enfermagem em Centro Cirúrgico do Hospital de Clínicas de Porto Alegre. Autora.

2 Professora Auxiliar de Ensino da Escola de Enfermagem da Universidade Federal do Rio Grande do Sul, Enfermeira da Sala de Recuperação Pós-Anestésica do Hospital de Clínicas de Porto Alegre.

3 Enfermeira da Sala de Recuperação Pós-Anestésica do Hospital de Clínicas de Porto Alegre.

4 Psicóloga Clínica da Unidade de Pediatria do Hospital de Clínicas de Porto Alegre.
} 
ração da família e de seu ambiente, para qualquer forma de tratamento.

Muitas vezes as crianças têm fantasias e imagens muito ruins a respeito das rotinas hospitalares, médicas e da equipe em geral. Alguns fatores necessitam ser examinados quando o enfermeiro se defrontar com estes pacientes, principalmente aqueles que submeterão a processos dolorosos e desagradáveis como as intervenções cirúrgicas.

Para a maioria dos autores, as crianças menores de cinco anos estão mais expostas aos ef eitos nefastos da hospitalização, pois são menos capazes de comunicar os seus desejos e têm uma concepção irreal da hospitalização, sendo, portanto, muito comum alguns comportamentos regressivos.

Para a criança cujo pensamento está em fase de evolução, a situação de hospitalização pode apresentar uma das oportunidades mais favoráveis para que ela recorra à fantasia, principalmente se não for devidamente esclarecida e apoiada.

MARTINS et alii (19797 referem que as crianças necessitam ser esclarecidas a respeito de futuros sofrimentos, porque a dor é sempre mais difícil de suportar quando chega de surpresa. Sabe-se, portanto, que o medo do desconhecido é uma das grandes fontes de ansiedade e sofrimento para o indivíduo.

Para GROHS (1984) as hospitalizações poderão repercutir na personalidade em formação das crianças e estas experiências estão sujeitas a algumas variáveis como o tempo de hospitalização, o grau de afastamento da mãe, tanto físico quanto afetivo, a idade em que incidiu, as diferenças individuais e o preparo prévio da criança e seus pais.

No papel ampliado do enfermeiro esforçando-se para prestar uma assistência globalizada à criança e sua família, é importante avaliar as respostas comportamentais da criança quando esta experimenta uma situação de stress. Deste modo os objetivos da assistência de enfermagem devem estar voltados para facilitar o comportamento de adaptação frente a uma situação agressiva para a criança, que é o ato cirúrgico, diminuindo os riscos de traumas e promovendo melhores condições de recuperação.

Os enfermeiros da sala de recuperação pós-anestésica (SRPA) do Hospital de Clínicas de Porto Alegre (HCPA) com o intuito de melhor assistir os pacientes do setor observaram que existia uma diferença no atendimento perstado nas unidades pediátricas e a SRPA e verificaram que esta diferença era relacionada com a recreação, ambiente físico e equipe.

Inicialmente estes enfermeiros realizaram reuniões entre todos os membros da equipe multidisciplinar que atendem as crianças no centro cirúrgico. Este encontro teve como objetivo traçar metas para organizar uma nova assistência às crianças na sala de recuperação.

0 trabalho da equipe multiprofissional, como ressaltam vários autores HARUNARI (1974), KRON (1978),
FERNANDES (1981) e ALVARENGA (1973) é fundamental para o sucesso de qualquer proposta de atendimento individualizado e globalizado à criança, pois é necessário a estreita colaboração entre todos os membros da equipe para que a criança perceba uma uniformidade de ações e sentimentos.

Observou-se que crianças quando são manipuladas indiscriminadamente por vários membros de uma mesma equipe técnica, sem que lhes dêem nenhuma satisfação sobre o que irá acontecer, são mais arredias ou rebeldes, dificultando o estabelecimento de um novo padrão de relacionamento.

Os enfermeiro da unidade, frente a estas observações, solicitaram à administração do HCPA que fossem feitas algumas modificações na área física da sala de recuperação determinada a receber as crianças. Reformas tais como melhora na iluminação do local e confecções de cortinas que impedissem às crianças visualizarem a área de adultos, entre outras.

GROHS (1984) salienta que "desde a entrada, o hospital infantil deve dar a impressão de acolhedor e descontraído, paredes coloridas, bonecas, salas de jogos, etc.".

Outra iniciativa do grupo de enfermeiros para melhorar o atendimento à criança na SRPA foi um treinamento teórico específico a todos os funcionários do setor no atendimento à criança numa unidade de bloco cirúrgico.

Segundo DRAIN \& SHIPPLEY (1981) na SRPA o ingrediente mais importante para uma assistência de enfermagem bem sucedida é uma equipe bem treinada, altamente habilitada. 0 cuidado de enfermagem deve ser centrado na pessoa do paciente, sendo o mesmo considerado como um indivíduo dependente de enfermagem devido ao seu estado de inconsciência ou de semi-consciência.

Em relação à permanência das crianças no período de recuperação pós-anestésica os enfermeiros observaram que elas ficavam muito paradas, apresentavam-se queixosas e sonolentas, sem terem alternativas de alguma atividade para fazer. Apesar do procedimento cirúrgico realizado, observava-se também que as mães tinham uma tendência a estimular as queixas das crianças e torná-las mais apáticas e queixosas.

KLEIN (1970) verificou que o brinquedo tem uma função de alívio para criança ao permitir a expressão de ansiedade, fantasias e sentimentos.

FREUD (1969) formulou, em 1920, em seu estudo "Mais Além do Princípio do Prazer", algumas características do jogo infantil, enfatizando que a criança obtém através dele, a transformação do passivo em ativo e a vivência de domínio de experiência traumática.

LEBOVICI \& DIATKINE (1985) sintetizaram a importância do brinquedo dizendo que o "trabalho está para o adulto assim como o brinquedo está para a criança". Isto quer dizer que enquanto o paciente brinca ex- 
pressa os seus sentimentos conseguindo muitas vezes falar sobre seus temores. Sabe-se que existem várias maneiras pelas quais a criança poderá alcançar a elaboração destas situações difíceis; poderá mudar os papéis, ou seja, onde foi agredido pode se transformar em agressora ou modificar a resolução desta situação providenciando um "final feliz" para um episódio que lhe foi desagradável. A maneira como cada criança reage frente a uma mesma situação traumática, depende, primeiramente de características individuais e a sua interação com o meio ambiente. Referem também que o modo como a criança brinca é um indicativo de como ela está, de como ela é.

As considerações apresentadas e o contato com alguns trabalhos realizados sobre o assunto é que levaram as autoras a considerar sumamente importante buscar meios de melhorar as relação equipe de enfermagem e criança e com isto tentar diminuir os traumas que uma experiência cirúrgica possa desencadear na criança. Como recurso para ajudar um melhor relacionamento com a equipe de enfermagem e ao mesmo tempo melhorar a mobilização da criança fazendo com que elas retornem mais rapidamente ao seu ambiente, introduziu-se há um ano na SRPA. Para isto, a equipe de enfermeiros solicitou o auxílio das psicólogas clínicas do HCPA.

Os brinquedos colocados na SRPA procuram atender as diversas faixas etárias e proporcionam ao paciente momentos em que, pela sua utilização ele possa elaborar de maneira mais adequada, isto é, com menos medo o processo de recuperação pós-cirúrgica imediato.

A partir do exposto tem-se como objetivos neste trabalho:

a) Estudar a influência do brinquedo no processo de recuperação da criança após um procedimento cirúrgico;

b) Comparar o comportamento das crianças que não recebem o estímulo brinquedo com o comportamento daquelas que recebem o estímulo brinquedo;

c) Comparar a média de permanência dos dois grupos.

\section{MATERIAL E MÉTODO}

O caráter da pesquisa é experimental e seguiu os seguintes passos metodológicos: características do campo de pesquisa, população e amostra do estudo, instrumento da pesquisa, procedimentos e tratamento estatístico.

\section{Características do Campo de Pesquisa}

0 presente trabalho foi realizado no HCPA, o qual é um hospital geral de capacidade extra e de corpo clínico fechado. É um hospital de ensino e serve de área hospitalar e de saúde pública para Escola de Enferma- gem e a Faculdade de Medicina da Universidade Federal do Rio Grande do Sul (UFRGS).

O Serviço de Enfermagem em Centro Cirúrgico é composto de quatro unidades; Unidade de Bloco $\mathrm{Ci}$ rúrgico (UBC), Unidade de Centro Cirúrgico Ambiental (UCCA), Unidade de Hemodinãmica (HD) e Centro de Material e Esterilização (CME).

O Bloco Cirúrgico, uma das unidades do Serviço de Enfermagem em Centro Cirúrgico, é constituído de dez salas de cirurgia e sala de recuperação pós-anestésica com capacidade para quinze leitos para adultos e cinco leitos para crianças atendendo pacientes provenientes das unidades de internação e de ambulatório.

A SRPA tem como rotina a permanência dos familiares junto às crinças durante o período de permanência dos mesmos, sendo que para as cirurgias ambulatoriais a presença do acompanhante torna-se obrigatória.

A equipe de enfermagem deste setor é composta por enfermeiros, auxiliares e atendentes abrangendo todos os turnos. A assistência de enfermagem prestada às crianças é de responsabilidade do enfermeiro $\mathrm{e}$ do auxiliar de enfermagem.

Cabe a o enfermeiro a coordenação da assistência, a prestação de cuidados mais complexos após uma rigorosa avaliação das condições gerais do paciente, $\mathrm{e}$ aos auxiliares a observação da criança e cuidados simples.

\section{População e Amostra do Estudo}

A população em estudo é constituída de pacientes pediátricos submetidos à cirurgias eletivas ambulatoriais na UBC do HCPA. A escolha destes pacientes deve-se ao fato de que a observação da influência do brinquedo poderia ser melhor evidenciado. As crianças que anteriormente estiveram hospitalizadas nas unidades de internação do HCPA já tiveram contato com uma equipe de enfermagem, mesmo que não seja a da SRPA. Receberam juntamente com seus pais uma orientação feita pelo enfermeiro sobre a cirurgia e além disto, participaram do programa de recreação que conta com a presença de psicólogos, terapeuta ocupacional e recreacionistas e, quando necessário, conta com 0 acompanhamento individual dos psicólogos. As cirurgias em pacientes hospitalizados são geralmente de grande porte e apresentam uma recuperação pós-operatóra diferente.

0 presente estudo, de caráter preliminar, contou com uma amostra que compreende trinta e quatro (34) crianças, de ambos os sexos, na faixa etária de zero a doze (12) anos, submetidas a um procedimento cirúrgico de pequeno e médio porte, e que para tanto sofreram anestesia geral.

Dividiu-se a amostra em dois grupos assim denominados:

GRUPO A - Dezessete (17) crianças que não rece- 
beram o brinquedo como estímulo durante o período de recuperação anestésica.

BRUPO B - Dezessete (17) crianças que receberam o brinquedo como estímulo durante o período de recuperação anestésica.

Foram utilizados como critérios para a seleção da amostra:

- ausência de complicações cirúrgica-anestésicas no trans e pós-operatório imediato;

- ausência de retardo mental.

\section{Instrumento da Pesquisa}

Empregou-se o método de observação e o registro foi feito num formulário elaborado especificamente para este fim.

0 formulário foi testado anteriormente em vinte e cinco (25) crianças e a partir da análise dos resultados obtidos foi elaborado o instrumento definitivo constituído de itens de identificação e itens de observação de comportamento.

Foram consideradas variáveis independentes para o presente estudo as categorias sexo, idade, tipos de cirurgia, de anestesia e tempo de permanência na SRPA. As variáveis dependentes constituíram-se de comportamentos apresentados pelas crianças na presença e ausência do estímulo brinquedo. Os comportamentos observados foram quanto à orientação, calma, confiança, estado de humor, receptividade e atenção, os quais foram conceituados pela equipe.

\section{Procedimentos}

\section{Coleta de Dados}

A coleta dos dados foi realizada pelas autoras do trabalho através de observação direta da criança feita ao longo da sua permanência na SRPA. Esta observação foi feita simultaneamente por duas das autoras e caso houvesse discordância nas anotações das reações apresentadas pelas crianças, utilizava-se uma terceira pessoa para que se chegasse a uma única definição.

Antes as autoras definiram as possíveis reações do comportamento das crianças a serem observadas com a finalidade de obterem uma uniformidade no levantamento dos dados.

\section{Seleção dos Brinquedos}

Para seleção e introdução dos brinquedos na SRPA foram considerados os aspectos que envolvem a assistência de enfermagem e aqueles relacionados com a assistência psicológica.

Nos aspectos relacionados com a assistência de enfermagem foram considerados como importantes o uso de material plástico como predominante, pois permite a limpeza e previne o risco de transmissão de microorganismos; formato do brinquedo de modo a prevenir acidentes ou ferimentos durante o manuseio, evitando-se objetos com pontas ou cantos pontiagudos.

Nos aspectos psicológicos foram considerados os tipos adequados de brinquedos de acordo com os interesses de cada faixa etária. Como nosso objetivo era fazer com que através da atividade lúdica, as crianças elaborassem a situação que estavam vivenciando, a equipe incluiu alguns materiais hospitalares, como gaze, máscaras, seringas plásticas e outros, de maneira que com o seu manuseio houvesse uma familiarização com esses objetos e tornasse mais fácil tolerar alguns procedimentos técnicos.

Foram utilizados brinquedos completamente prontos (carrros, bonecos, etc.) e outros que exigem criatividade das próprias crianças (quebra-cabeças e materiais de jogos como papel, lápis de colorir).

\section{Tratamento Estatístico dos Dados}

0 tratamento estatístico foi realizado através da distribuição dos dados em freqüências absolutas e ferqüências relativas.

Para análise comparativa foi aplicado o teste " $\mathrm{T}$ " de STUDENT para duas Amostras Independentes.

\section{APRESENTAC̣ÃO E ANÁLISE DOS RESULTADOS}

Apresenta-se a seguir a análise dos resultados obtidos a partir do tratamento manual e eletrônico dos dados.

Quanto às variáveis independentes, a amostra evidenciou os seguintes achados:

Destaca-se nesta Tabela a predominância do sexo masculino em ambos os grupos.

TABELA 1 - Distribuição das crianças dos dois grupos estudados, segundo sexo.

\begin{tabular}{|c|c|c|c|c|c|c|}
\hline GRUPOS & \multicolumn{2}{|c|}{ SEM BRINQUEDOS } & \multicolumn{2}{|c|}{ COM BRINQUEDOS } & \multicolumn{2}{|c|}{ TOTAL } \\
\hline SEXO & CASOS & $\%$ & CASOS & $\%$ & CASOS & $\%$ \\
\hline Masculino & 13 & 76,5 & 11 & 64,7 & 24 & 70,6 \\
\hline Feminino & 4 & 23,5 & 6 & 35,3 & 10 & 29,4 \\
\hline TOTAL & 17 & 100,0 & 17 & 100,0 & 34 & 100,0 \\
\hline
\end{tabular}


TABELA 2 - Distribuição das crianças dos dois grupos estudados segundo idade.

\begin{tabular}{|c|c|c|c|c|}
\hline \multirow{2}{*}{$\begin{array}{l}\text { GRUPO } \\
\text { IDADE (ANOS) }\end{array}$} & \multicolumn{2}{|c|}{ SEM BRINQUEDO } & \multicolumn{2}{|c|}{ COM BRINQUEDO } \\
\hline & CASOS & $\%$ & CASOS & $\%$ \\
\hline $0 \longmapsto 2$ & 0 & 0,0 & 6 & 35,3 \\
\hline $2 \longmapsto 5$ & 9 & 52,9 & 6 & 35,3 \\
\hline $5 \longmapsto 8$ & 4 & 23,5 & 4 & 23,5 \\
\hline $8 \longmapsto 11$ & 2 & $\begin{array}{l}20,0 \\
11,8\end{array}$ & 1 & 5,9 \\
\hline $11 \longmapsto 14$ & 2 & 11,8 & 0 & 0,0 \\
\hline TOTAL & 18 & 100,0 & 17 & 100,0 \\
\hline
\end{tabular}

Observa-se nesta Tabela que nas faixas etárias dos dois grupos houve diferença na população nos extremos.

TABELA 3 - Distribuição das crianças dos dois grupos estudados segundo o tempo de permanência na sala de recuperação pós-anestésica.

\begin{tabular}{c|c}
\hline SEM BRINQUEDOS & COM BRINQUEDOS \\
\hline $45 \mathrm{~min}$ & $25 \mathrm{~min}$ \\
$110 \mathrm{~min}$ & $80 \mathrm{~min}$ \\
$110 \mathrm{~min}$ & $75 \mathrm{~min}$ \\
$110 \mathrm{~min}$ & $110 \mathrm{~min}$ \\
$150 \mathrm{~min}$ & $70 \mathrm{~min}$ \\
$155 \mathrm{~min}$ & $35 \mathrm{~min}$ \\
$220 \mathrm{~min}$ & $160 \mathrm{~min}$ \\
$120 \mathrm{~min}$ & $225 \mathrm{~min}$ \\
$220 \mathrm{~min}$ & $30 \mathrm{~min}$ \\
$205 \mathrm{~min}$ & $30 \mathrm{~min}$ \\
$120 \mathrm{~min}$ & $200 \mathrm{~min}$ \\
$135 \mathrm{~min}$ & $190 \mathrm{~min}$ \\
$125 \mathrm{~min}$ & $195 \mathrm{~min}$ \\
$148 \mathrm{~min}$ & $135 \mathrm{~min}$ \\
$280 \mathrm{~min}$ & $155 \mathrm{~min}$ \\
$250 \mathrm{~min}$ & $120 \mathrm{~min}$ \\
$305 \mathrm{~min}$ & $255 \mathrm{~min}$ \\
\hline TOTAL 2800 & TOTAL \\
\hline
\end{tabular}

Sem Brinquedos - Média de permanência em min. igual a 165 ' $\left(2 \mathrm{~h} 455^{\prime}\right)$

Com Brinquedos - Média de permanência em min. igual a 135' (2h30')

Conclui-se que estatisticamente a diferença não é si gnificativa para $\mathrm{P}>0,05$.

Teste T Student para duas Amostras Independentes.

TABELA 4 - Distribuição das crianças dos dois grupos estudados segundo os coportamentos finais.

\begin{tabular}{l|cr|rr}
\hline \multicolumn{1}{c|}{ COMPORTAMENTO } & \multicolumn{2}{c}{ SEM BRINQUEDO } & \multicolumn{2}{c}{ COM BRINQUEDO } \\
& CASOS & $\%$ & CASOS \\
\hline Orientado & 16 & 94,1 & 17 & 100,0 \\
Calmo & 13 & 76,5 & 15 & 88,2 \\
Confiante & 7 & 41,2 & 13 & 76,5 \\
Alegre & 4 & 23,5 & 9 & 52,9 \\
Receptivo & 10 & 58,8 & 14 & 82,4 \\
Atento & 15 & 88,2 & 17 & 100,0 \\
\hline
\end{tabular}


Admitindo-se a $\mathrm{P}=0,05$ no teste de Fisher para a significãncia constata-se que o brinquedo não afetou significativamente o comportamento das crianças pois a partir deste teste obtivemos o valor de $\mathrm{P} \quad 0,05$ para a maioria dos comportamentos. Porém para o comportamento "Confiante" houve significãncia estatística $(\mathrm{P}<0,05)$, contudo atribuindo-se a $\mathrm{P}=0,06$ como valor constata-se que caso a amostra fosse maior talvez mostrasse alguma diferença significativa.

\section{DISCUSSÃO DOS RESULTADOS}

No que diz respeito ao comportamento final das crianças constata-se que o grupo que utilizou brinquedos teve um comportamento mais positivo em relação à orientação, tranqüilidade, confiança, alegria, receptividàde e atenção do que o grupo controle, evidenciado através da comparação das freqüências relativas de ambos os grupos.

Conforme ANGERAMI et alii (1984) e HARUNARI (1974), a recreação constitui um excelente meio para canalizar de maneira positiva as variáveis do desenvolvimento da criança para uma educação integral. Em se tratando de criança hospitalizada, neutralizar os fatores de ordem psicológica, que naturalmente surgem com a doença.

Observa-se que os itens de orientação, tranqüilidade e atenção tiveram em seu conjunto menor diferença de.freqüência entre os dois grupos.

Por outro lado, destacam-se os dados referentes aos itens de confiança, alegria e receptividade demonstrando uma maior diferença entre as freqüências relativas favorável ao grupo de crianças com brinquedo. Cabe, aqui, ressaltar a importância destes itens no comportamento da criança, por se tratarem de reações que exigem da criança maior interação com o meio externo, com outras pessoas e maior envolvimento afetivo.

Estes dados vão ao encontro da afirmação de WAECHTER (1979) o brinquedo terapêutico é destinado a auxiliar as crianças a desenvolver sua capacidade de relacionar-se com a realidade externa, estabelecer uma ponte entre o hospital e o ambiente externo e desenvolver um sentido de unidade dentro de si mesmas. A superação das experiências traumáticas durante a hospitalização poderá ser favorecida pela concessão de oportunidades para a criança "brincar" com estas experiências, porque freqüentemente elas não podem verbalizá-las ou pensar nelas através de outros, como poderiam as crianças maiores e os adultos.

Este resultado ainda torna-se mais representativo ao se analisar a diferença na distribuição da amostra com relação às faixas etárias, ao indicar que as crianças do grupo com brinquedo, de menor faixa etária, apresentaram uma resposta mais positiva no relacionamento com o meio e com a equipe de saúde demonstrado através da alegria, confiança e receptividade.
O item confiança estatisticamente significativo, estabelece que a criança ao se relacionar melhor com a equipe de enfermagem, interagiu bem com o local (até então desconhecido) facilitando a prestação de cuidados necessários nesta situação e talvez diminuindo o tempo de permanência.

Tendo em vista a idade das crianças estudadas e a menor faixa etária do grupo com brinquedo, tem-se que salientar a participação dos pais na recuperação dos pacientes. Pode-se observar que o comportamento das crianças durante a sua estada na sala de recuperação estava estreitamente ligada à maneira como estes se comportavam frente aos filhos.

Além da idade das crianças e da influência dos pais vários outros fatores podem interferir no comportamento das crianças, como as experiências prévias, a informação dos pais e da criança a respeito da cirurgia, a história pregressa da criança e as características pessoais de cada uma.

Dos 34 casos estudados, vinte e uma crianças acordaram agitadas e destes pacientes seis continuaram com este comportamento apesar da entrada da mãe na sala de recuperação. Pode-se, talvez inferir que estes seis pacientes não interagiram de uma maneira positiva com seus familiares e que a maneira como enfrentaram o processo cirúrgico causou-lhes um stress muito grande, provavelmente devido às dificultades anteriores com seus pais.

As informações que a equipe presta aos familiares vão ser benéficas na medida em que conseguem auxiliar as crianças e elucidar dúvidas e fantacia. Na maioria das vezes, quando os pais tiveram uma experiência negativa de hospitalização ou de intervenções cirúrgicas transmitem para as crianças o sentimento de que estes momentos não foram bons.

Em alguns casos estudados os pais relataram que o retardo na realização dos procedimentos cirúrgicos, ora realizados, foi devido à dificuldade de tolerar que seus filhos tivessem que vivenciar estas situações traumáticas.

A criança, principalmente a de menor faixa etária, quando predomina o pensamento mágico a nível de fantasia, sente a cirurgia como uma punição e isso é confirmado na medida em que ela não tem de seus pais o apoio emocional suficiente.

Observa-se, na prática, que a sintomatologia psicológica negativa no pós-operatório tem uma distribuição por idade, correspondendo a maior freqüência de sintomas em crianças abaixo de 3 anos. Após esta idade, observou-se uma diminuição dos mesmos.

Em relação ao brinquedo o principal objetivo é fazer com que a criança brinque realmente, consiga efeitos, construa, faça combinações e crie. Os brinquedos devem na sua maioria, reproduzir de uma forma fidedigna a realidade. Um exemplo disso são alguns brinquedos adotados neste estudo, que reproduzem objetos ligados a ambiente hospitalar e terapêutico, co- 
mo estetoscópios, seringas, aparelhos de pressão, etc. É muito importante que a criança saiba qual é a utilidade do brinquedo e que ao brincar leve este jogo ao seu final, cuidando deste material lúdico.

Segundo AJURIAGUERRA (1983), o modo de relação criança-médico é definido de maneira extremamente ambígua pela criança e ela expressa, com freqüência através do "brincar de médico" ou pelos desenhos realizados. Neste brinquedo nitidamente observa-se que o médico representa o papel paterno que assume características de potência e que inspira, ao mesmo tempo, medo. Isto fica mais evidente, quando se trata de uma experiência cirúrgica, onde o cirurgião que é vivido pela criança como um agressor ao mesmo tempo é uma figura que precisa ser respeitada não podendo ser alvo de agressões provenientes das crianças, pois sentem que dependem muito deles para o seu bem-estar. O papel materno é mais fácil de evidenciar, sendo geralmente bom e é representado com freqüência pelo pael de enfermeiro quando, quando cria um ambiente delicado e acolhedor.

Observou-se ainda no brinquedo e nas brincadeiras um interesse muito grande de todos as crianças, nos desenhos, nos brinquedos que imitam materiais hospitalares e em bonecos da atualidade (HULK, HE-MAN, HOMEM ARANHA, etc...).

No que se refere aos desenhos, as crianças representam praticamente as situações vivenciadas. Nesta atividade lúdica, leva-se em conta a cor, o tamanho e os personagens representados sendo de grande valia os diálogos referidos pelas crianças enquanto desenham.

Em relação ao tempo de permanência das crianças na sala de recuperação constatou-se uma redução de 30 ' no grupo de pacientes com brinquedos se comparado com o grupo controle. Este resultado analisado através do teste T de Student para duas Amostras Independentes não se torna estatisticamente significativo. Porém, considerando que a média de permanência do total da amostra foi de 2:30' (150 min) houve uma diminuição de $20 \%$ do tempo de recuperação das crianças que receberam o estímulo do brinquedo.

A importãncia deste resultado torna-se mais evidente se comparado a média de permanência das crianças há 1 ano atrás, antes do setor sofrer qualquer influência de modificação para 0 atendimento às crianças (treinamento da equipe de enfermagem e reformas da área). Nesta época a média de permanência era de 3h11' (211 min) havendo uma redução deste tempo de $21,81 \%$ no grupo sem brinquedos e de $36,02 \%$ no grupo com brinquedo.

Frente a estas considerações observa-se que as modificações introduzidas na unidade como treinamento de pessoal, modificações na área física e, principalmente, a introdução de brinquedos tiveram influência marcante na redução do tempo de permanência dos pacientes na SRPA.
Para SMITH (1979), o tempo de permanência da criança na sala de recuperação pós-anestésica está na razão direta com o tempo de permanência da criança na sala de cirurgia. Quanto mais demora o ato cirúrgico mais tempo levará a criança para recuperar-se da anestesia e ir para casa.

\section{CONSIDERAC̣ÕES FINAIS}

Cabe destacar que dada a limitação da amostra os achados aqui arrolados não são passíveis de generalização, aplicando-se apenas aos grupos estudados. Não se pode, entretanto, esquecer que mensurar emoções é tarefa difícil, uma vez que as mesmas se prestam mais à qualificação do que à quantificação, pois emanam do ser humano.

Para tanto os autores pretendem continuar os estudos à respeito da influência do brinquedo nas criarças na SRPA, pois é possível que uma observação mais longa e detida venham alterar ou ratificar os achados deste estudo.

\section{CONCLUSÕES}

Neste trabalho, em vista de todas as discussões e considerações que foram feitas, chegou-se às seguintes conclusões:

a) do ponto de vista quantitativo para se poder afirmar que há a influência do brinquedo no processo de recuperação da criança, após um procedimento cirúrgico as autoras acreditam que a amostra estudada deverá ser aumentada;

b) o brinquedo incluído no processo de recuperação da criança trouxe um comportamento final mais positivo;

c) a média de permanência sof reu uma redução de 30 minutos naquele tempo que teve o estímulo de brinquedos.

\section{REFERÊNCIAS BIBLIOGRÁFICAS}

1. AJURIAGUERRA, J. Manual de psiquiatria infantil. Paris, Masson, 1983

2.ALVARENGA, Adelaide. A necessidade multiprofissional para o melhor atendimento hospitalar. Revista Paulista de Hospitais. São Paulo, 21 (1): 36-8, jan., 1973.

3. ANGERAMI, V. A. et alii. Psicologia hospitalar: a atuação do psicólogo no contexto hospitalar. São Paulo, Ed. Traço, 1984.

4. DRAIN, C. B. \& SHIPLEY,S.B. Enfermagem na sala de recuperação. Rio de Janeiro, Ed. Interamericana, 1981.

5. FERNANDES, J. D. Contribuição da equipe multiprofissional nas ações de saúde: mito ou realidade? Revista Brasileira de Enfermagem, Brasília, 34 (2): 175-81, abr/maio/jun., 1981.

6. FREUD, Sigmund. Mais além do princípio do prazer. Edição Standard. Rio de Janeiro, Imago, 1969.

7. GROHS, G. e. M. Hospitalização de crianças. Revista Psiquiatria do RGS, 6 (3) 173-77, set./dez., 1984. 
8. HARUNARI, Luiza. Recreação infantil em hospital. São Paulo, 1974.

9. KLEIN, Melanie. Contribuições à psicanálise. São Paulo, Ed. Mestreson, 1970.

10. KRON, T. Manual de enf ermagem. 4. ed. Rio de Janeiro, Interamericana, 1978. $251 \mathrm{p}$.

11. LEBOVICI \& DIATKINE. Significado e função do brinquedo na criança. Porto Alegre, Artes Médicas, 1985.
12. MARTINS, Dyrce Maria R. et alii. Assistência de enfermagem a crianças hospitalizadas quando enfrentam situações desagradáveis. Revista Escola Enfermagem da USP, São Paulo, ago. 1979.

13. SMITH, Robert M. Anesthesia for infants and children. 4. ed., London, Mosby, 1979.

14. WAETCHER, Eugênia H. \& BLAKE, Florence G. Enf ermagem pediátrica. 9. ed., Rio de Janeiro, Interamericana, 1979. 